\title{
iccafd
}

Revista Iberoamericana de Ciencias de la Actividad Fisica y el Deporte

\section{FISIOTERAPIA Y SU ROL EN EL ALTO RENDIMIENTO: UNA REVISIÓN SISTEMÁTICA DE LA LITERATURA}

\section{PHYSIOTHERAPY AND HIS ROLE IN HIGH PERFORMANCE: A SYSTEMATIC REVIEW OF LITERATURE}

\section{Alfonso Mantilla, José Iván}

José Iván Alfonso Mantilla. Fisioterapeuta, Universidad del Rosario, Colombia, Josealfonso25@hotmail.com

Código UNESCO: 321311 . Fisioterapia

Clasificación Consejo de Europa: 14. Fisioterapia y Rehabilitación

Recibido el 24 de octubre de 2017

Aceptado el 23 de enero de 2018

Correspondencia:

José Iván Alfonso Mantilla

Josealfonso25@hotmail.com

\section{RESUMEN}

El deporte de alto rendimiento, exige que se desarrollen estrategias de recuperación, entrenamiento y rehabilitación que favorezcan la adaptación al entrenamiento físico. En fisioterapia, se usan estrategias para mantener la salud del deportista en su más alto nivel y entre las estrategias más utilizadas se encuentran prevención de lesiones, entrenamiento funcional, recuperación funcional enfocados en objetivos específicos del deportista. Por tal motivo, el objetivo de este artículo es realizar una revisión sistemática de la literatura sobre el rol del fisioterapeuta en el deporte de alto rendimiento. rendimiento

PALABRAS CLAVE: rehabilitación, fisioterapia, medicina deportiva, alto

\section{ABSTRACT}

High-performance sport requires the development of recovery, training and rehabilitation strategies that favor adaptation to physical training. In physiotherapy, strategies are used to maintain the health of the athlete at the highest level, among the strategies most used are injury prevention, functional training, functional recovery focused on specific goals of the athlete. For this reason, the objective of this article is to perform a systematic review of the literature on the role of physiotherapist in high performance sport.

KEY WORDS: rehabilitation, physical therapy, sports medicine, athletic performance. 


\section{INTRODUCCIÓN}

La fisioterapia es el área de estudio del movimiento corporal humano, esta se enfatiza en el estudio de alteraciones que afecten la funcionalidad del ser humano en aspectos de la vida diaria. Las alteraciones del movimiento corporal humano pueden estar dadas por enfermedades y lesiones a nivel osteomuscular, neuromuscular y cardiovascular(1). A nivel deportivo, la fisioterapia estudia la relación del cuerpo humano con el ejercicio físico. Por ejemplo, el fisioterapeuta entiende la relación de un proceso de preparación física en alto rendimiento con factores como nutrición, recuperación y entrenamiento para alcanzar el máximo potencial del deportista y llevarlo al éxito deportivo(2). En el alto rendimiento, el fisioterapeuta es un miembro dentro de un equipo interdisciplinar que garantiza el adecuado estado físico de un deportista. De hecho, la fisioterapia se encarga de realizar atención a deportistas con lesiones a nivel osteomuscular y neuromuscular, realizar programas de prevención de lesiones, entrenamiento de cualidades físicas para retorno a práctica deportiva, programas de recuperación después de la realización de ejercicio físico de alta intensidad. Por tal motivo, la fisioterapia se ha convertido en una de las áreas de estudio en ciencias de la salud que garantiza la adaptación y recuperación del movimiento corporal humano en el área deportiva(3-11).

Las lesiones deportivas afectan la funcionalidad del deportista llevándolo a alterar sus actividades de la vida diaria, las lesiones pueden producirse en cualquier momento del día del deportista y tienen diferentes factores etiológicos como sexo, nivel de competición, tipo de deporte, factores intrínsecos y extrínsecos. Las lesiones pueden darse en diferentes deportes como futbol, judo, balonmano, baloncesto y voleibol reportándose en mayor medida lesiones de cuello de pie como esguinces, rodilla como ruptura de ligamento cruzado anterior y posterior, lesiones de cabeza y cara, muñeca, espalda baja, hombro(12-18). La fisioterapia es una de las áreas de la salud que trabaja en el deporte de alto rendimiento con el fin de garantizar que el deportista se encuentre en óptimas condiciones para afrontar competencias a nivel nacional e internacional. El fisioterapeuta dentro de un equipo deportivo realiza trabajos como prevención de lesiones, recuperación, rehabilitación y entrenamiento. Por ejemplo, el desarrollo de circuitos funcionales enfocados en la prevención demuestran tener efectos significativos en la reducción de lesiones deportivas, estos circuitos se basan principalmente en habilidades propioceptivas, potencia, coordinación, velocidad, resistencia y fuerza(19-23). En segunda instancia, la fisioterapia realiza los trabajos de recuperación de fatiga muscular inducida por la concentración de cargas de entrenamiento a través de herramientas como hidratación, crioterapia, electroestimulación(24-27). Es por eso que el objetivo de este artículo es realizar una revisión sistemática de la literatura sobre el rol del fisioterapeuta en el deporte de alto rendimiento. 


\section{MATERIAL Y MÉTODOS}

Se realizo una revisión sistemática de la literatura. Se determinaron los siguientes criterios de inclusión y exclusión:

Inclusión: Estudios contemplados del año 2000 al 2017, literatura que contemplara los siguientes términos MeSH: Rehabilitation, Physical therapy, sports medicine, athletic performance. La literatura se aceptaría en idioma español, ingles y portugués

Se estableció como otro criterio de inclusión que la búsqueda de evidencia se basara en: Fuentes primarias: Estudios ECA, Metaanálisis, casos y controles, estudios de cohorte, revisiones sistemáticas, revisiones de literatura. Fuentes secundarias: Monografías, tesis de grado, libros.

Exclusión: Estudios que su año de publicación fuera inferior al año 2000, que no contemplaran los términos $\mathrm{MeSH}$ establecidos, estudios que no estuvieran disponibles o completos

\section{RESULTADOS}

En la figura 1 se muestra el proceso de extracción de la evidencia identificada en las bases de datos y su proceso de selección.

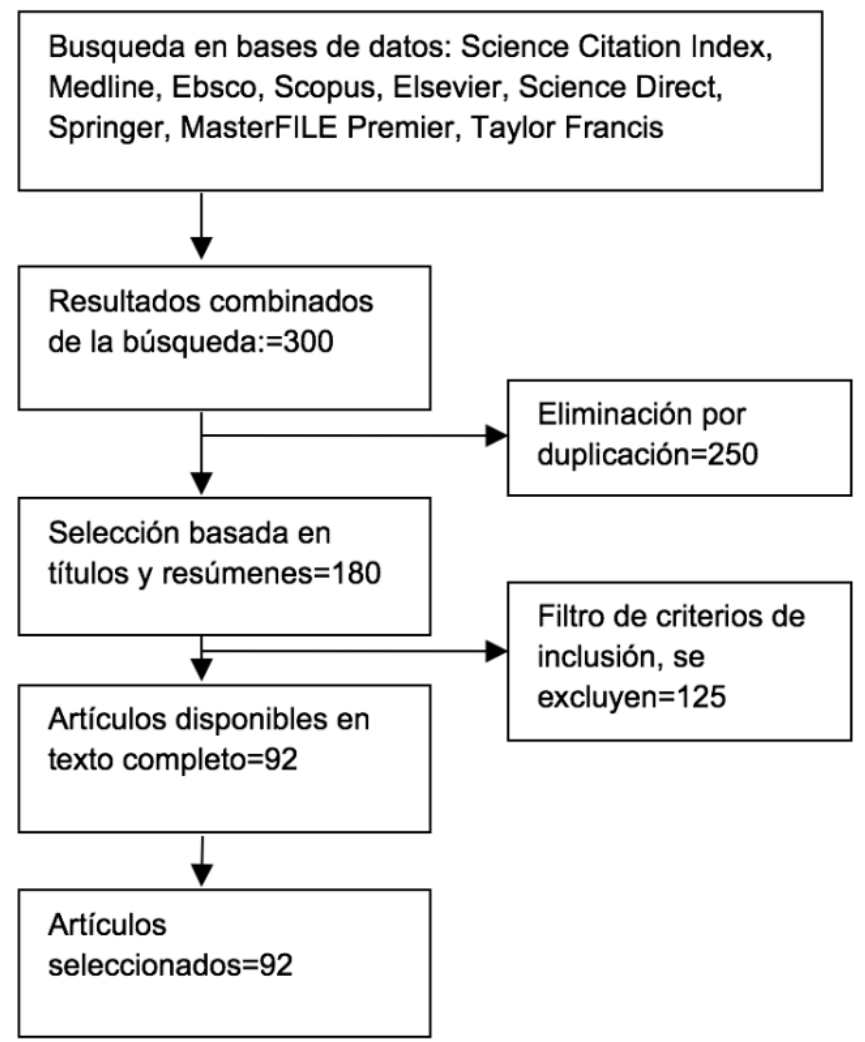

Figura 1. (Flujograma de extracción de la evidencia). Elaboración propia, 2016. 
En los artículos se pudo evidenciar que el fisioterapeuta es un profesional esencial en los equipos deportivos debido a que es el responsable de la ejecución de actividades enfocadas en la salud del deportista. Dentro de la revisión se pudieron identificar actividades que realiza el fisioterapeuta para mantener en óptimas condiciones a los deportistas y los principios para realizar una adecuada planificación de procesos de rehabilitación deportiva.

\section{Planificación de procesos de rehabilitación deportiva (Habilidades paramétricas y sinergistas)}

Desde fisioterapia, se ha desarrollo un concepto de rehabilitación basado en habilidades especificas del movimiento corporal humano, este concepto es aplicado en procesos de rehabilitación de lesiones con el fin de brindar una proceso de adaptación global en el paciente. Para ejemplificar, la rehabilitación debe basarse desde el componente motor y sensorial. En primera instancia, el componente motor tiene una teoría que tiene como punto de partida una pirámide en la cual la base es conocida como habilidades paramétricas las cuales están conformadas por resistencia, velocidad, fuerza las cuales son esenciales para la adaptación de trabajos específicos; la segunda base conocida como habilidades sinergistas se encuentra la activación reciproca y en la punta de la pirámide las habilidades compuestas donde se combinan los elementos de la primera y segunda base produciendo habilidades complejas como coordinación, equilibrio, velocidad y balance las cuales son el punto esencial de trabajo de circuitos integrados de rehabilitación específicos en el gesto motor. El componente sensorial se basa en el trabajo conjunto de los sistemas vestibular, propioceptivo y visual para trabajar todas las habilidades del movimiento corporal humano(28).

\section{Programas de prevención de lesiones}

El fisioterapeuta trabaja en conjunto con diversas áreas de la salud dentro de los equipos deportivos con el fin de mantener al deportista sano y en óptimas condiciones para afrontar futuras competencias. Por ejemplo, en los equipos deportivos se trabaja en conjunto con preparación física en el desarrollo de circuitos funcionales de prevención de lesiones, los cuales integren elementos del movimiento corporal humano tales como coordinación, propiocepción, equilibrio, core, fuerza concéntrica y excéntrica, resistencia y potencia. Estos circuitos han demostrado reducir significativamente las lesiones deportivas en los equipos. Estos circuitos de entrenamiento se pueden realizar dos veces por semana por un periodo de duración de 20 minutos a través de ejercicios funcionales de movimiento utilizando elementos como bancos, bases inestables, maquina de fuerza, bandas elásticas. Adicionalmente, estos circuitos trabajan los elementos centrales y periféricos del movimiento corporal humano obteniendo un balance a nivel articular, muscular, biomecánico y sensorial(2023, 29-33). 


\section{Recuperación funcional del deportista}

La fatiga es un síntoma constante en el deportista de alto rendimiento, está en mayor medida se produce por las cargas de entrenamiento impuestas durante las jornadas de entrenamiento, viajes a competencias nacionales e internacionales, alteración del ciclo del sueño, inadecuada alimentación(34). El equipo médico es participe de los procesos de recuperación garantizando el estado físico de los jugadores, se utilizan distintas herramientas para garantizar la recuperación. En primera instancia, se encuentra la crioterapia como medio de recuperación de fatiga muscular e inflamación producida por cargas de entrenamiento, esta es prescrita de distintas formas. La más común es inmersión con un tiempo de aplicación de 20 minutos a una temperatura promedio de $5{ }^{\circ} \mathrm{C}$.

Otra forma es la criocámara la cual utiliza temperaturas de recuperación en promedio de $-60^{\circ} \mathrm{C} \mathrm{a}-110^{\circ} \mathrm{C}$ por un periodo de tiempo de 3 minutos(35-42). En segunda instancia, se encuentran los baños de contraste son una herramienta que al igual que la crioterapia reduce el dolor, edema, espasmos musculares y acelera la demanda metabólica para ayudar a la recuperación muscular esta se realiza con contrastes entre medios físicos calientes y fríos $(43,44)$. En tercera instancia, la electroestimulación muscular se ha convertido en una herramienta por excelencia de recuperación, se han creado distintos aparatos que manejan corrientes específicas de recuperación muscular de fatiga y dolor este tratamiento puede ser usado durante un tiempo de 15- 30 minutos(27, 45-48).

De igual manera, existen otros métodos como los medios de compresión estos pueden usarse a través de ropa y medios neumáticos, estos medios han demostrado índices significativos de disminución del dolor muscular, fatiga muscular, marcadores bioquímicos como la creatina quinasa y la proteína $C$ reactiva relacionadas con el sobrentrenamiento y fatiga muscular(49-55). La liberación miofascial se ha utilizado como un medio de recuperación en alto rendimiento, esta se realiza con ayuda de rollos terapéuticos con diferentes densidades para realizar un masaje sobre la zona para liberar el tejido adherido(56-61). El masaje deportivo también se utiliza para garantizar procesos de recuperación, existen distintas técnicas para realizar el masaje en la cual se distinguen la presión, fuerza, velocidad de aplicación sobre los vientres musculares, este puede tener una duración de 10 minutos mejorando el dolor y fatiga muscular(62-67). El estiramiento es otra herramienta fundamental para realizar procesos de recuperación muscular después de que realizan cargas de entrenamiento altas, se pueden usar estiramientos activos o estáticos(68-71). El plasma rico en plaquetas es una herramienta utilizada para acelerar la recuperación utilizando factores de crecimiento y proteínas que aceleran y estimulan la regeneración tisular de huesos, músculos, cartílagos y tendones. Adicionalmente, en combinación con programas de rehabilitación específicos demuestran tener mayor efectividad y acelerar el tiempo de recuperación de lesiones musculares que en el deporte profesional es de vital importancia(72-78). La hidratación es un 
factor fundamental de recuperación y de prevención de lesiones deportivas debido a que se debe tener una adecuada homeostasis corporal después de la perdida de fluidos a consecuencia de la realización de actividad física, mantener una adecuada hidratación a nivel muscular garantiza menor tasa de lesiones deportivas. Por ejemplo, una inadecuada hidratación en altas temperaturas puede causar una alteración del rendimiento deportivo e hiponatremia, el sodio es un factor importante en la recuperación y gasto urinario. Adicionalmente, los carbohidratos aumentan la oxidación de la glucosa y mantienen la energía utilizada durante la práctica deportiva lo cual garantiza un adecuado rendimiento del deportista(79-83).

\section{Rehabilitación funcional en el deporte}

La fisioterapia es parte fundamental de los procesos de recuperación de lesiones deportivas, estos profesionales utilizan diferentes elementos y herramientas para realizar la rehabilitación de lesiones deportivas y poder devolver al jugador al campo, la rehabilitación deportiva se basa en principios de fases las cuales se deben seguir para obtener procesos exitosos. Adicionalmente, se deben guiar por protocolos y guías como parte del proceso de rehabilitación. Los procesos de rehabilitación deben garantizar restaurar el rango de movimiento, balance intrarticular, simetría muscular, fuerza muscular, estabilidad dinámica, control neuromuscular como elementos base del movimiento corporal humano(84-89). La rehabilitación debe ser un proceso secuencial respetando los periodos de curación de los tejidos para así garantizar un adecuado resultado. De igual manera, se deben utilizar todas las herramientas para brindar al deportista el mejor proceso y garantizar un trabajo global enfocándose en elementos como estabilidad, control postural, potencia, resistencia, agilidad, coordinación y técnica a través de pruebas especificas del gesto motor del deporte(90-92).

\section{DISCUSIÓN}

Con los datos obtenidos se pudo evidenciar que el fisioterapeuta es un profesional que comprende las lesiones de los sistemas musculoesqueléticos, cardiovascular y neuromuscular. Los fisioterapeutas son los encargados de llevar a cabo los procesos de recuperación de deportistas de alto rendimiento, estos conocen de forma exacta los mecanismos de lesión, anatomía, biomecánica, tiempo de recuperación de los tejidos para brindarle al deportista una recuperación adecuada en casa fase de la rehabilitación. Adicionalmente, estos están presentes en todo el tiempo de recuperación y son un apoyo incondicional para el deportista lo cual es un factor adicional para la consecución de objetivos del deportista. La rehabilitación no solo se basa en curar el origen de la enfermedad sino de potencializar y mejorar las demás condiciones físicas del deportista durante su proceso de rehabilitación, es por eso de vital importancia que siempre se le exija al deportista durante el proceso respetando los límites del mismo. En la actualidad, se cuenta con gran número de evidencia científica con respecto al tema de lesiones deportivas y su rehabilitación, de igual manera se puede encontrar guías y protocolos de manejo para diferentes tipos 
de lesiones. Es por eso, que todos los fisioterapeutas deben conocer la evidencia actual y guiarse por protocolos estandarizados a nivel mundial y tener una posición crítica frente a la evidencia para realizar mejores procesos de planificación y metodología de rehabilitación. En segunda instancia, el fisioterapeuta dentro de un equipo deportivo además de participar en los procesos de recuperación es participe en el trabajo interdisciplinar para la creación de circuitos funcionales de prevención de lesiones que han demostrado reducir significativamente el riesgo de lesiones en el deportista, deben crearse circuitos basados en habilidades básicas de movimiento con el fin de perfeccionar dichas habilidades, los ejercicios deben hacerse en circuitos que garanticen el trabajo de los sistemas garantes de movimiento. En tercera instancia, los trabajos de recuperación deben realizarse basados en estándares y evidencia científica para garantizar la supercompesación del deportista con el fin de que día a día pueda entrenarse de la mejor manera y aumentar sus cualidades físicas durante los microciclos de entrenamiento.

La fisioterapia es un área en constante cambio, las investigaciones a nivel mundial generan conocimiento que debe ser analizado por profesionales para garantizar su aplicación en poblaciones específicas. Es por eso, que en fisioterapia la investigación debe ser un punto de partida para la creación de nuevos sistemas de intervención, rehabilitación y análisis del movimiento corporal humano.

\section{LIMITACIONES Y CAMINOS FUTUROS}

En Colombia, se ha demostrado que el deporte es un pilar para el desarrollo social de su población, en la última década se ha visto un aumento en la consagración de títulos a nivel suramericano, panamericano y olímpico en distintas disciplinas deportivas. Es por eso, que las ramas de salud debemos trabajar para garantizar el estado optimo de deportistas para afrontar estas competencias. En especial, los fisioterapeutas colombianos deben trabajar en mayor medida por desarrollar investigaciones en el área de prevención y recuperación de deportistas nacionales con el fin de ampliar el conocimiento a nivel nacional para crear protocolos nacionales estandarizados para la intervención con distintos tipo de disciplinas deportivas. Es por eso que desde etapas estudiantiles debe educarse al estudiante sobre la importancia y necesidad de la investigación como camino al desarrollo de nuevas intervenciones y posicionamiento del gremio

\section{CONCLUSIONES}

En conclusión, el rol del fisioterapeuta en alto rendimiento se basa en acciones tales como prevención de lesiones, hidratación deportiva, planificación y metodología de procesos de rehabilitación y recuperación funcional de deportistas. Adicionalmente, el fisioterapeuta cumple un rol de educador en salud con el fin de enseñar al deportista las herramientas para el cuidado de la aptitud física con para preparación para competencias nacionales e internacionales. 


\section{CONFLICTO DE INTERESES}

El autor declara que no existe conflicto de intereses.

\section{REFERENCIAS}

1. Lesmes JD. Evaluación clínico-funcional del movimiento corporal humano: Ed. Médica Panamericana; 2007.

2. Dolores JCLM. Intervención clínica en deporte de alto rendimiento: una experiencia en fútbol sala. Cuadernos de Psicología del Deporte. 2005;5.

3. Athanasopoulos S, Kapreli E, Tsakoniti A, Karatsolis K, Diamantopoulos K, Kalampakas K, et al. The 2004 Olympic Games: physiotherapy services in the Olympic Village polyclinic. Br J Sports Med. 2007;41(9):603-9; discussion 9.

4. Grant ME, Steffen K, Glasgow P, Phillips N, Booth L, Galligan M. The role of sports physiotherapy at the London 2012 Olympic Games. Br J Sports Med. 2014;48(1):63-70.

5. Phillips N. Measuring physiotherapy intervention in high performance sport. Phys Ther Sport. 2014;15(1):1-2.

6. Zainuddin Z, Newton M, Sacco P, Nosaka K. Effects of massage on delayedonset muscle soreness, swelling, and recovery of muscle function. J Athl Train. 2005;40(3):174-80.

7. Peake JM, Roberts LA, Figueiredo VC, Egner I, Krog S, Aas SN, et al. The effects of cold water immersion and active recovery on inflammation and cell stress responses in human skeletal muscle after resistance exercise. J Physiol. 2016.

8. Cervera FJA, Rotger MAN. El fisioterapeuta en la prevención de lesiones del deporte. Revista de fisioterapia. 2006;5(2):31-6.

9. LaStayo PC, Woolf JM, Lewek MD, Snyder-Mackler L, Reich T, Lindstedt SL. Eccentric muscle contractions: their contribution to injury, prevention, rehabilitation, and sport. J Orthop Sports Phys Ther. 2003;33(10):557-71.

10. Middleton $P$, Montero C. [Eccentric muscular contraction: implications in treatment of athletes]. Ann Readapt Med Phys. 2004;47(6):282-9.

11. Setuain I, Izquierdo M, Idoate F, Bikandi E, Gorostiaga EM, Aagaard P, et al. Differential Effects of Two Rehabilitation Programs Following Anterior Cruciate Ligament Reconstruction. J Sport Rehabil. 2016:1-37.

12. Henke T, Luig P, Schulz D. [Sports injuries in German club sports, Aspects of epidemiology and prevention]. Bundesgesundheitsblatt Gesundheitsforschung Gesundheitsschutz. 2014;57(6):628-37.

13. Beynnon BD, Vacek PM, Murphy D, Alosa D, Paller D. First-time inversion ankle ligament trauma: the effects of sex, level of competition, and sport on the incidence of injury. Am J Sports Med. 2005;33(10):1485-91.

14. Reckling C, Zantop T, Petersen W. [Epidemiology of injuries in juvenile handball players]. Sportverletz Sportschaden. 2003;17(3):112-7.

15. Junge A, Dvorak J. Soccer injuries: a review on incidence and prevention. Sports Med. 2004;34(13):929-38.

16. Peterson L, Junge A, Chomiak J, Graf-Baumann T, Dvorak J. Incidence of football injuries and complaints in different age groups and skill-level groups. Am J Sports Med. 2000;28(5 Suppl):S51-7.

17. Bromley SJ, Drew MK, Talpey S, Mclntosh AS, Finch CF. A systematic review of prospective epidemiological research into injury and illness in Olympic combat sport. $\mathrm{Br}$ J Sports Med. 2017.

18. Indriethadottir MH, Sveinsson T, Magnusson K, Arngrimsson SA, Johannsson E. [Prevalence of sport injuries, sport participation and drop out due to injury in young adults]. Laeknabladid. 2015;101(10):451-6. 
19. Faude $O$, Rossler $R$, Junge $A$. Football injuries in children and adolescent players: are there clues for prevention? Sports Med. 2013;43(9):819-37.

20. Barengo NC, Meneses-Echavez JF, Ramirez-Velez R, Cohen DD, Tovar G, Bautista JE. The impact of the FIFA 11+ training program on injury prevention in football players: a systematic review. Int J Environ Res Public Health. 2014;11(11):11986-2000. 21. Silvers-Granelli HJ, Bizzini M, Arundale A, Mandelbaum BR, Snyder-Mackler L. Does the FIFA 11+ Injury Prevention Program Reduce the Incidence of ACL Injury in Male Soccer Players? Clin Orthop Relat Res. 2017.

22. Bizzini M, Dvorak J. FIFA 11+: an effective programme to prevent football injuries in various player groups worldwide-a narrative review. Br J Sports Med. 2015;49(9):5779.

23. Bizzini M, Junge A, Dvorak J. Implementation of the FIFA 11+ football warm up program: how to approach and convince the Football associations to invest in prevention. Br J Sports Med. 2013;47(12):803-6.

24. Russell M, Birch J, Love T, Cook CJ, Bracken RM, Taylor T, et al. The Effects of a Single Whole-Body Cryotherapy Exposure on Physiological, Performance, and Perceptual Responses of Professional Academy Soccer Players After Repeated Sprint Exercise. J Strength Cond Res. 2017;31(2):415-21.

25. Rowsell GJ, Coutts AJ, Reaburn P, Hill-Haas S. Effects of cold-water immersion on physical performance between successive matches in high-performance junior male soccer players. J Sports Sci. 2009;27(6):565-73.

26. Vanderthommen M, Makrof S, Demoulin C. Comparison of active and electrostimulated recovery strategies after fatiguing exercise. J Sports Sci Med. 2010;9(2):164-9.

27. Finberg M, Braham R, Goodman C, Gregory P, Peeling P. Effects of electrostimulation therapy on recovery from acute team-sport activity. Int $\mathrm{J}$ Sports Physiol Perform. 2013;8(3):293-9.

28. Carr JH, Shepherd RB. Neurological Rehabilitation: Optimizing Motor Performance: Churchill Livingstone; 2010.

29. Steffen K, Emery CA, Romiti M, Kang J, Bizzini M, Dvorak J, et al. High adherence to a neuromuscular injury prevention programme (FIFA 11+) improves functional balance and reduces injury risk in Canadian youth female football players: a cluster randomised trial. Br J Sports Med. 2013;47(12):794-802.

30. Longo UG, Loppini M, Berton A, Marinozzi A, Maffulli N, Denaro V. The FIFA 11+ program is effective in preventing injuries in elite male basketball players: a cluster randomized controlled trial. Am J Sports Med. 2012;40(5):996-1005.

31. Ejnisman B, Barbosa G, Andreoli CV, de Castro Pochini A, Lobo T, Zogaib R, et al. Shoulder injuries in soccer goalkeepers: review and development of a FIFA 11+ shoulder injury prevention program. Open Access J Sports Med. 2016;7:75-80.

32. Al Attar WS, Soomro N, Pappas E, Sinclair PJ, Sanders RH. How Effective are F-MARC Injury Prevention Programs for Soccer Players? A Systematic Review and Meta-Analysis. Sports Med. 2016;46(2):205-17.

33. Meurer MC, Silva MF, Baroni BM. Strategies for injury prevention in Brazilian football: Perceptions of physiotherapists and practices of premier league teams. Phys Ther Sport. 2017;28:1-8.

34. Kovacs MS, Baker LB. Recovery interventions and strategies for improved tennis performance. Br J Sports Med. 2014;48 Suppl 1:i18-21.

35. Crystal NJ, Townson DH, Cook SB, LaRoche DP. Effect of cryotherapy on muscle recovery and inflammation following a bout of damaging exercise. Eur J Appl Physiol. 2013;113(10):2577-86.

36. Hohenauer E, Taeymans J, Baeyens JP, Clarys P, Clijsen R. The Effect of PostExercise Cryotherapy on Recovery Characteristics: A Systematic Review and MetaAnalysis. PLoS One. 2015;10(9):e0139028. 
37. Fonda B, Sarabon N. Effects of whole-body cryotherapy on recovery after hamstring damaging exercise: a crossover study. Scand J Med Sci Sports. 2013;23(5):e270-8.

38. Ferreira-Junior JB, Bottaro M, Vieira CA, Soares SR, Vieira A, Cleto VA, et al. Effects of partial-body cryotherapy $(-110$ degrees $C$ ) on muscle recovery between highintensity exercise bouts. Int J Sports Med. 2014;35(14):1155-60.

39. Kruger M, de Marees M, Dittmar KH, Sperlich B, Mester J. Whole-body cryotherapy's enhancement of acute recovery of running performance in well-trained athletes. Int J Sports Physiol Perform. 2015;10(5):605-12.

40. Poppendieck W, Faude O, Wegmann M, Meyer T. Cooling and performance recovery of trained athletes: a meta-analytical review. Int J Sports Physiol Perform. 2013;8(3):227-42.

41. Costello JT, Baker PR, Minett GM, Bieuzen F, Stewart IB, Bleakley C. Wholebody cryotherapy (extreme cold air exposure) for preventing and treating muscle soreness after exercise in adults. Cochrane Database Syst Rev. 2015(9):Cd010789.

42. Bleakley C, McDonough S, Gardner E, Baxter GD, Hopkins JT, Davison GW. Cold-water immersion (cryotherapy) for preventing and treating muscle soreness after exercise. Cochrane Database Syst Rev. 2012(2):Cd008262.

43. Malanga GA, Yan N, Stark J. Mechanisms and efficacy of heat and cold therapies for musculoskeletal injury. Postgrad Med. 2015;127(1):57-65.

44. Petrofsky JS, Khowailed IA, Lee H, Berk L, Bains GS, Akerkar S, et al. Cold Vs. Heat After Exercise-Is There a Clear Winner for Muscle Soreness. J Strength Cond Res. 2015;29(11):3245-52.

45. Beaven CM, Cook C, Gray D, Downes P, Murphy I, Drawer S, et al. Electrostimulation's enhancement of recovery during a rugby preseason. Int $\mathrm{J}$ Sports Physiol Perform. 2013;8(1):92-8.

46. Erten YT, Sahinkaya T, Dinc E, Kilinc BE, Bayraktar B, Kurtoglu M. The effects of compression garments and electrostimulation on athletes' muscle soreness and recovery. J Exerc Rehabil. 2016;12(4):308-13.

47. Grunovas A, Silinskas V, Poderys J, Trinkunas E. Peripheral and systemic circulation after local dynamic exercise and recovery using passive foot movement and electrostimulation. J Sports Med Phys Fitness. 2007;47(3):335-43.

48. Taylor T, West DJ, Howatson G, Jones C, Bracken RM, Love TD, et al. The impact of neuromuscular electrical stimulation on recovery after intensive, muscle damaging, maximal speed training in professional team sports players. J Sci Med Sport. 2015;18(3):328-32.

49. Armstrong SA, Till ES, Maloney SR, Harris GA. Compression socks and functional recovery following marathon running: a randomized controlled trial. J Strength Cond Res. 2015;29(2):528-33.

50. Hill J, Howatson G, van Someren K, Leeder J, Pedlar C. Compression garments and recovery from exercise-induced muscle damage: a meta-analysis. Br J Sports Med. 2014;48(18):1340-6.

51. Armstrong SA, Till ES, Maloney S, Harris G. Compression socks and functional recovery following marathon running: a randomised controlled trial. J Strength Cond Res. 2014.

52. Hill JA, Howatson G, van Someren KA, Walshe I, Pedlar CR. Influence of compression garments on recovery after marathon running. J Strength Cond Res. 2014;28(8):2228-35.

53. Overmayer RG, Driller MW. Pneumatic Compression Fails to Improve Performance Recovery in Trained Cyclists. Int J Sports Physiol Perform. 2017:1-21.

54. Martin JS, Friedenreich ZD, Borges AR, Roberts MD. Acute Effects of Peristaltic Pneumatic Compression on Repeated Anaerobic Exercise Performance and Blood Lactate Clearance. J Strength Cond Res. 2015;29(10):2900-6. 
55. Cochrane DJ, Booker HR, Mundel T, Barnes MJ. Does intermittent pneumatic leg compression enhance muscle recovery after strenuous eccentric exercise? Int J Sports Med. 2013;34(11):969-74.

56. Cheatham SW, Kolber MJ, Cain M, Lee M. The effects of self-myofascial release using a foam roll or roller massager on joint range of motion, muscle recovery, and performance: a systematic review. Int J Sports Phys Ther. 2015;10(6):827-38.

57. Schroeder AN, Best TM. Is self myofascial release an effective preexercise and recovery strategy? A literature review. Current sports medicine reports. 2015;14(3):2008.

58. Pearcey GE, Bradbury-Squires DJ, Kawamoto J-E, Drinkwater EJ, Behm DG, Button DC. Foam rolling for delayed-onset muscle soreness and recovery of dynamic performance measures. Journal of athletic training. 2015;50(1):5-13.

59. Arroyo-Morales M, Olea N, Martínez MM, Hidalgo-Lozano A, Ruiz-Rodríguez C, Díaz-Rodríguez L. Psychophysiological effects of massage-myofascial release after exercise: a randomized sham-control study. The journal of alternative and complementary medicine. 2008;14(10):1223-9.

60. Healey KC, Hatfield DL, Blanpied P, Dorfman LR, Riebe D. The effects of myofascial release with foam rolling on performance. The Journal of Strength \& Conditioning Research. 2014;28(1):61-8.

61. Best TM, Hunter R, Wilcox A, Haq F. Effectiveness of sports massage for recovery of skeletal muscle from strenuous exercise. Clin J Sport Med. 2008;18(5):44660.

62. Delextrat A, Calleja-Gonzalez J, Hippocrate A, Clarke ND. Effects of sports massage and intermittent cold-water immersion on recovery from matches by basketball players. J Sports Sci. 2013;31(1):11-9.

63. Poppendieck W, Wegmann M, Ferrauti A, Kellmann M, Pfeiffer M, Meyer T. Massage and Performance Recovery: A Meta-Analytical Review. Sports Med. 2016;46(2):183-204.

64. Paoli A, Bianco A, Battaglia G, Bellafiore M, Grainer A, Marcolin G, et al. Sports massage with ozonised oil or non-ozonised oil: Comparative effects on recovery parameters after maximal effort in cyclists. Phys Ther Sport. 2013;14(4):240-5.

65. Ali Rasooli S, Koushkie Jahromi M, Asadmanesh A, Salesi M. Influence of massage, active and passive recovery on swimming performance and blood lactate. $J$ Sports Med Phys Fitness. 2012;52(2):122-7.

66. Moraska A. Sports massage. A comprehensive review. J Sports Med Phys Fitness. 2005;45(3):370-80.

67. Fuller JT, Thomson RL, Howe PR, Buckley JD. Vibration Therapy Is No More Effective Than the Standard Practice of Massage and Stretching for Promoting Recovery From Muscle Damage After Eccentric Exercise. Clin J Sport Med. 2015;25(4):332-7.

68. Torres R, Pinho F, Duarte JA, Cabri JM. Effect of single bout versus repeated bouts of stretching on muscle recovery following eccentric exercise. J Sci Med Sport. 2013;16(6):583-8.

69. Chen $\mathrm{CH}$, Chen TC, Jan MH, Lin JJ. Acute effects of static active or dynamic active stretching on eccentric-exercise-induced hamstring muscle damage. Int J Sports Physiol Perform. 2015;10(3):346-52.

70. Torres R, Ribeiro F, Alberto Duarte J, Cabri JM. Evidence of the physiotherapeutic interventions used currently after exercise-induced muscle damage: systematic review and meta-analysis. Phys Ther Sport. 2012;13(2):101-14.

71. Guzel Y, Karalezli N, Bilge O, Kacira BK, Esen H, Karadag H, et al. The biomechanical and histological effects of platelet-rich plasma on fracture healing. Knee Surg Sports Traumatol Arthrosc. 2015;23(5):1378-83.

72. Grambart ST. Sports medicine and platelet-rich plasma: nonsurgical therapy. Clin Podiatr Med Surg. 2015;32(1):99-107. 
73. Sanchez M, Delgado D, Sanchez P, Fiz N, Azofra J, Orive G, et al. Platelet rich plasma and knee surgery. Biomed Res Int. 2014;2014:890630.

74. Pourcho AM, Smith J, Wisniewski SJ, Sellon JL. Intraarticular platelet-rich plasma injection in the treatment of knee osteoarthritis: review and recommendations. Am J Phys Med Rehabil. 2014;93(11 Suppl 3):S108-21.

75. MS AH, Mohamed Ali MR, Yusof A, George J, Lee LP. Platelet-rich plasma injections for the treatment of hamstring injuries: a randomized controlled trial. Am J Sports Med. 2014;42(10):2410-8.

76. Arnoczky SP, Sheibani-Rad S. The basic science of platelet-rich plasma (PRP): what clinicians need to know. Sports Med Arthrosc. 2013;21(4):180-5.

77. Kon E, Filardo G, Di Martino A, Marcacci M. Platelet-rich plasma (PRP) to treat sports injuries: evidence to support its use. Knee Surg Sports Traumatol Arthrosc. 2011;19(4):516-27.

78. Zoorob R, Parrish ME, O'Hara H, Kalliny M. Sports nutrition needs: before, during, and after exercise. Prim Care. 2013;40(2):475-86.

79. Kalman DS, Feldman S, Krieger DR, Bloomer RJ. Comparison of coconut water and a carbohydrate-electrolyte sport drink on measures of hydration and physical performance in exercise-trained men. J Int Soc Sports Nutr. 2012;9(1):1.

80. Rehrer NJ. Fluid and electrolyte balance in ultra-endurance sport. Sports Med. 2001;31(10):701-15.

81. Kenefick RW, Cheuvront SN. Hydration for recreational sport and physical activity. Nutr Rev. 2012;70 Suppl 2:S137-42.

82. Shirreffs SM, Sawka MN. Fluid and electrolyte needs for training, competition, and recovery. J Sports Sci. 2011;29 Suppl 1:S39-46.

83. Mantilla JIA, Santa JM. Circuitos funcionales en rehabilitación. Movimiento Científico. 2015;9(2):61-9.

84. Dreinhofer KE, Schuler S, Schafer M, Ohly T. [Rehabilitation concepts and return to sport after interventions on the shoulder]. Orthopade. 2014;43(3):256-64.

85. Fournier M. Principles of rehabilitation and return to sports following injury. Clin Podiatr Med Surg. 2015;32(2):261-8.

86. Yabroudi MA, Irrgang JJ. Rehabilitation and return to play after anatomic anterior cruciate ligament reconstruction. Clin Sports Med. 2013;32(1):165-75.

87. Liem BC, Truswell HJ, Harrast MA. Rehabilitation and return to running after lower limb stress fractures. Curr Sports Med Rep. 2013;12(3):200-7.

88. Mithoefer K, Hambly K, Logerstedt D, Ricci M, Silvers H, Della Villa S. Current concepts for rehabilitation and return to sport after knee articular cartilage repair in the athlete. J Orthop Sports Phys Ther. 2012;42(3):254-73.

89. Myer GD, Paterno MV, Ford KR, Quatman CE, Hewett TE. Rehabilitation after anterior cruciate ligament reconstruction: criteria-based progression through the returnto-sport phase. J Orthop Sports Phys Ther. 2006;36(6):385-402.

90. Nedelec M, McCall A, Carling C, Legall F, Berthoin S, Dupont G. Recovery in soccer: part I - post-match fatigue and time course of recovery. Sports Med. 2012;42(12):997-1015.

91. Nedelec M, McCall A, Carling C, Legall F, Berthoin S, Dupont G. Recovery in soccer : part ii-recovery strategies. Sports Med. 2013;43(1):9-22.

Referencias totales citadas: 91

Referencias citadas correspondientes a la Rev Ib CC Act Fis Dep: 1 